\section{(6) OPEN ACCESS}

- Additional material is published online only. To view please visit the journal online (http://dx.doi.org/10.1136/ gutjnl-2014-308653).

For numbered affiliations see end of article.

\section{Correspondence to} Dr Michael Goggins, Johns Hopkins Medical Institutions, Department of Pathology, 1550 Orleans Street, Baltimore, MD 21231, USA; mgoggins@jhmi.edu

Received 16 October 2014 Revised 26 November 2014 Accepted 19 December 2014 Published Online First 30 January 2015

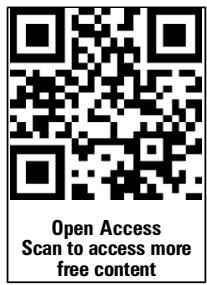

\section{SLinked}

- http://dx.doi.org/10.1136/ gutjnl-2014-309066

\title{
Time to progression of pancreatic ductal adenocarcinoma from low-to-high tumour stages
}

\author{
Jun Yu, ${ }^{1}$ Amanda L Blackford, ${ }^{2}$ Marco dal Molin, ${ }^{1}$ Christopher L Wolfgang, ${ }^{1,2,3}$ \\ Michael Goggins ${ }^{1,2,4}$
}

\section{ABSTRACT}

Objective Although pancreatic ductal adenocarcinoma is considered a rapidly progressive disease, mathematical models estimate that it takes many years for an initiating pancreatic cancer cell to grow into an advanced stage cancer. In order to estimate the time it takes for a pancreatic cancer to progress through different tumor, node, metastasis (TNM) stages, we compared the mean age of patients with pancreatic cancers of different sizes and stages.

Design Patient age, tumour size, stage and demographic information were analysed for 13131 patients with pancreatic ductal adenocarcinoma entered into the National Cancer Institute's Surveillance, Epidemiology and End Results (SEER) database. Multiple linear regression models for age were generated, adjusting for patient ethnicity, gender, tumour location and neoplastic grades.

Results African-American ethnicity and male gender were associated with an earlier age at diagnosis. Patients with stage I cancers (mean age 64.8 years) were on average 1.3 adjusted years younger at diagnosis than those with stage IV cancers $(p=0.001$ ). Among patients without distant metastases, those with T1 stage cancers were on average 1.06 and 1.19 adjusted years younger, respectively, than patients with T3 or T4 cancers ( $p=0.03$ for both). Among patients with stage IIB cancers, those with T1/T2 cancers were 0.79 adjusted years younger than those with T3 cancers ( $p=0.06)$. There was no significant difference in the mean adjusted age of patients with stage IA versus stage IB cancers.

Conclusions These results are consistent with the hypothesis that once pancreatic ductal adenocarcinomas become detectable clinically progression from low-stage to advanced-stage disease is rapid.

\section{INTRODUCTION}

Patients with pancreatic ductal adenocarcinoma usually present with advanced-stage cancers, have rapidly progressive disease and a poor prognosis. For this reason, pancreatic screening studies have evaluated the utility of using endoscopic ultrasound and MRI/MR cholangiopancreatography-based screening for individuals with a family history of pancreatic cancer in multiple first-degree relatives to try and detect asymptomatic early pancreatic cancers and precancerous lesions. ${ }^{1-9}$ There is a high prevalence of precancerous lesions among patients who meet criteria for undergoing pancreatic screening. ${ }^{1}$ These lesions include intraductal papillary mucinous neoplasms (IPMNs) typically identified as

\section{Significance of this study}

What is already known on this subject?

- Evidence based on mutational analysis predicts that pancreatic cancers are confined to the pancreas for many years, perhaps a decade or more before spreading beyond the pancreas.

- Clinical experience indicates that most patients with advanced stage pancreatic cancer progress rapidly.

- Certain patient characteristics are associated with an earlier average age of pancreatic cancer diagnosis, such as cigarette smoking and African-American ethnicity.

What are the new findings?

- We find, after adjusting for factors associated with age at diagnosis, the average age differences of patients with larger versus smaller T stage tumours is small and often not statistically significant, suggesting that most pancreatic cancers rapidly progress from lower to higher T stages.

- We estimate that the average T1-stage pancreatic cancer progresses to T4 stage in just over 1 year.

\section{How might it impact on clinical practice in} the foreseeable future?

- Recommendations for the surveillance of patients undergoing pancreatic screening need to take into account the potential for the rapid progression of pancreatic cancer.

- To avoid detecting late-stage cancers, early detection efforts have to focus on detecting and treating very small subcentimeter pancreatic cancers and carcinoma in situ.

pancreatic cysts, and pancreatic intraepithelial neoplasia (PanIN) generally identified only after pathological examination of the resected pancreas. ${ }^{10}$ Pancreatic screening protocols base their recommendations for surveillance ${ }^{11}$ largely on the characteristics of the pancreatic cysts identified by screening and on the overall experience of following patients with incidentally-detected pancreatic cysts, ${ }^{10}{ }^{12}$ despite evidence that most pancreatic cancers are thought to arise from undetected PanIN rather than from cysts, even in participants who are candidates for pancreatic screening. ${ }^{11}{ }^{13}$ An important goal of 
pancreatic screening is to identify and treat any pancreatic cancers that develop while they are still small and confined to the pancreas and ideally to identify and treat carcinoma in situ (ie, lining pancreatic cystic neoplasms or as PanIN-3 lesions) before invasive pancreatic cancer develops. ${ }^{11}{ }^{14}$ Pancreatic cysts with suspicious features can be imaged and sampled directly, but tests to detect PanIN-3 lesions such as by sampling secretin-stimulated pancreatic fluid are still investigational. ${ }^{15}$

Since pancreatic imaging tests cannot reliably detect PanIN lesions and can only detect a pancreatic cancer mass once it reaches several millimetres in diameter, ${ }^{16}$ it is important to understand the natural history of the early clinical stages of invasive pancreatic cancer. Reliable estimates of the time it takes pancreatic cancers to progress from its earliest detectable stage to more advanced stages are needed to determine surveillance intervals for patients undergoing pancreatic screening. A study using pancreatic cancer passenger mutations to model tumour progression concluded that pancreatic cancer remains confined to the pancreas for many years. ${ }^{17}$ This prediction contrasts with the experience of many patients who present with advanced-stage pancreatic cancer and have rapidly progressive disease. One reason for this apparent discrepancy is that this model estimates time starting from when the first cancer cell emerges until it progresses to an advanced cancer and includes the period when the cancer is too small $(<4-$ $5 \mathrm{~mm}$ in diameter) to be detectable by clinical tests. This undetectable period of growth may represent the majority of the overall time to progression, since a founding cancer cell has to undergo many tumour doublings to become a detectable tumour (a $1 \mathrm{~cm}$ diameter tumour contains $\sim 1$ billion cells), but only a few more doublings to grow into an advanced-stage tumour. The most relevant timeframe for early detection purposes is the time from when a cancer first becomes detectable by diagnostic tests up until it begins to progress beyond stage I disease. Although pancreatic cancer has a poor prognosis overall, patients with small stage I cancers have a better outcome than most patients with resectable cancers (mostly stage IIB). ${ }^{18-20}$

If it takes several years for most small early-stage clinically detectable pancreatic cancers to progress to advanced-stage disease, screening intervals can account for this and focus on trying to detect small stage I pancreatic cancers. On the other hand, if pancreatic cancers usually progress rapidly through its clinical stages (such as 1 year), then patients destined to develop pancreatic cancer are much more likely to present with advanced-stage cancers even if they undergo regular and frequent surveillance, unless screening protocols can reliably detect and treat preinvasive lesions.

Several studies have estimated the growth rate of various cancers using serial tumour marker measurements or serial CT scanning, ${ }^{21}$ but only limited data of this kind is available for pancreatic cancer. One study that measured serial serum CA19-9 and carcinoma embryonic antigen (CEA) measurements in patients with advanced stage disease estimated the average tumour doubling time of pancreatic cancers to be $\sim 40-$ 60 days. $^{22}$ Studies have attempted to estimate tumour growth using experimental models, ${ }^{23}$ but these models may not sufficiently represent the growth of primary human cancers.

If the average time required for pancreatic cancers to progress through different stages is long enough, it should be reflected in the average age of patients diagnosed at each stage of disease. In this study we analyse patient and tumour data in the National Cancer Institute's (NCI) Surveillance, Epidemiology and End Results (SEER) database to evaluate factors associated with age and tumour size at diagnosis and then used this information to compare the average age of patients with small and low-stage pancreatic cancers versus those with larger and higher stage cancers in order to estimate the average time it takes pancreatic cancer to progress through its clinical stages.

\section{PATIENTS AND METHODS}

We analysed the data of patients with pancreatic ductal adenocarcinoma entered into the NCI's SEER database, considered to be representative of the US population. ${ }^{24}$ In the SEER database, there were 13131 patients aged between 30 and 95 years and diagnosed with stage I to IV pancreatic ductal adenocarcinoma (American Joint Committee on Cancer (AJCC), Seventh edition, described in online supplementary table S1) between January 2004 and December 2011 who had all the required data for this study (demographics, primary site, tumour size, tumour stage, neoplastic grade, diagnostic confirmation, surgery of the primary site for patients with stage I and stage II disease). The SEER data includes patients from 18 registries: Alaska, Atlanta, Connecticut, California (excluding San Francisco, Los Angeles and San Jose), Detroit, Georgia (excluding Atlanta and rural Georgia), rural Georgia, Hawaii, Iowa, Kentucky, Los Angeles, Louisiana, New Jersey, New Mexico, San Francisco-Oakland SMSA, San Jose-Monterey, Seattle and Utah. We included cases with pancreatic ductal adenocarcinoma who had data available for all the following inclusion criteria: malignant behaviour, known age, age at diagnosis (codes: 30-115), race (codes: white, black, other), sex (codes: male and female), primary site (codes: C25.0-head of pancreas, C25.1-body of pancreas, C25.2-tail of pancreas), neoplastic grade (codes: well/moderately/poorly differentiated and undifferentiated), diagnostic confirmation, AJCC stage (codes: IA, IB, IIA, IIB, III and IV), tumour size (codes: 5-988), therapy; surgery of the primary site for patients with stage I and stage II disease (codes: 0-90). We excluded cases with variant histology such as those with cystic adenocarcinomas and neuroendocrine cancers.

\section{Statistics}

The primary goal of the analysis was to identify factors associated with age at diagnosis and use adjusted patient ages to estimate the average time to progression of pancreatic cancer through and within its clinical stages. Since primary tumour burden is more accurately quantified than the extent of metastatic disease, the analysis focused on estimating the average time to progression among patients who had localised or locally advanced disease. Patient and tumour characteristics associated with age at diagnosis were examined using student $t$ tests. Differences in age by patient groups defined by disease stage and tumour size were estimated with multivariable linear regression models that adjusted for sex, race, tumour location and grade. No hypotheses were defined a priori, and therefore $\mathrm{p}$ values are not adjusted for multiple comparisons and are presented for descriptive purposes only. Analyses were completed using SPSS (V.22.0) and R (V.3.1.1).

\section{RESULTS}

The patient characteristics associated with age at diagnosis in the SEER database are listed in tables 1 and 2. Males with pancreatic ductal adenocarcinoma were significantly younger than females (mean age (95\% CI), 65.2 (64.9 to 65.5 ) vs 66.8 (66.5 to 67.1) years; $\mathrm{p}<0.0001)$. Males also had slightly larger primary tumours on average than females (mean tumour diameter; 3.9 (3.9 to 4.0) vs $3.7 \mathrm{~cm}$ (3.7 to 3.8$)(\mathrm{p}<0.0001)$. African-Americans were also significantly younger at the time of diagnosis than Caucasians 
Table 1 Characteristics of all Surveillance, Epidemiology and End Results (SEER) participants, by their stage of pancreas cancer

\begin{tabular}{|c|c|c|c|c|c|c|}
\hline & $\begin{array}{l}\text { Stage IA } \\
N=409\end{array}$ & $\begin{array}{l}\text { Stage IB } \\
N=757\end{array}$ & $\begin{array}{l}\text { Stage IIA } \\
\mathrm{N}=1907\end{array}$ & $\begin{array}{l}\text { Stage IIB } \\
\mathrm{N}=5192\end{array}$ & $\begin{array}{l}\text { Stage III } \\
\mathrm{N}=1305\end{array}$ & $\begin{array}{l}\text { Stage IV } \\
\mathrm{N}=3561\end{array}$ \\
\hline Age at diagnosis-median (range) & $66(30,88)$ & $66(31,89)$ & $68(31,93)$ & $66(30,95)$ & $67(31,94)$ & $67(30,95)$ \\
\hline Age at diagnosis—mean (SD) & $64.8(11.1)$ & $64.9(12)$ & $67.1(10.6)$ & $65.7(10.9)$ & $66.1(11.5)$ & $66.1(12)$ \\
\hline \multicolumn{7}{|l|}{ Race-number (\%) } \\
\hline Black & 37 (9) & $88(12)$ & $177(9)$ & $462(9)$ & 177 (14) & 494 (14) \\
\hline Other & $29(7)$ & 69 (9) & $162(8)$ & $331(6)$ & $116(9)$ & $271(8)$ \\
\hline White & $343(84)$ & $600(79)$ & $1568(82)$ & $4399(85)$ & $1012(78)$ & $2796(79)$ \\
\hline \multicolumn{7}{|l|}{ Sex-number (\%) } \\
\hline Female & $201(49)$ & 395 (52) & $947(50)$ & 2537 (49) & $654(50)$ & $1646(46)$ \\
\hline Male & $208(51)$ & $362(48)$ & $960(50)$ & $2655(51)$ & $651(50)$ & $1915(54)$ \\
\hline \multicolumn{7}{|l|}{ Location—number (\%) } \\
\hline Body & $61(15)$ & $124(16)$ & $147(8)$ & $272(5)$ & $234(18)$ & $702(20)$ \\
\hline Head & $248(61)$ & $441(58)$ & $1469(77)$ & $4421(85)$ & 979 (75) & $1944(55)$ \\
\hline Tail & $100(24)$ & $192(25)$ & $291(15)$ & $499(10)$ & $92(7)$ & $915(26)$ \\
\hline \multicolumn{7}{|l|}{ Grade—no. (\%) } \\
\hline G1 & $174(43)$ & $208(27)$ & $254(13)$ & $564(11)$ & 197 (15) & $415(12)$ \\
\hline G2 & $172(42)$ & $359(47)$ & $982(51)$ & $2582(50)$ & $517(40)$ & $1182(33)$ \\
\hline G3 & $56(14)$ & $178(24)$ & $629(33)$ & $1966(38)$ & $563(43)$ & $1826(51)$ \\
\hline G4 & $7(2)$ & $12(2)$ & $42(2)$ & $80(2)$ & $28(2)$ & $138(4)$ \\
\hline Size $(\mathrm{cm})$ - median (range) & $1.5(0.5,2)$ & $3.2(2.1,15)$ & $3(0.7,14)$ & $3.3(0.5,15)$ & $4(0.5,14)$ & $4(0.5,15)$ \\
\hline \multicolumn{7}{|l|}{ Size $(\mathrm{cm})$ - number $(\%)$} \\
\hline$\leq 2 \mathrm{~cm}$ & $409(100)$ & $0(0)$ & $345(18)$ & 735 (14) & $93(7)$ & $234(7)$ \\
\hline $2-4 \mathrm{~cm}$ & $0(0)$ & $535(71)$ & $1095(57)$ & $3124(60)$ & $594(46)$ & 1554 (44) \\
\hline$>4 \mathrm{~cm}$ & $0(0)$ & $222(29)$ & $467(24)$ & $1333(26)$ & $618(47)$ & $1773(50)$ \\
\hline \multicolumn{7}{|l|}{ T stage—number (\%) } \\
\hline $\mathrm{T} 1$ & $409(100)$ & $0(0)$ & $0(0)$ & $172(3)$ & $0(0)$ & $111(3)$ \\
\hline $\mathrm{T} 2$ & $0(0)$ & $757(100)$ & $0(0)$ & $624(12)$ & $0(0)$ & $1070(30)$ \\
\hline $\mathrm{T} 3$ & $0(0)$ & $0(0)$ & $1907(100)$ & $4396(85)$ & $0(0)$ & $1514(43)$ \\
\hline T4 & $0(0)$ & $0(0)$ & $0(0)$ & $0(0)$ & $1305(100)$ & 866 (24) \\
\hline \multicolumn{7}{|l|}{$\mathrm{N}$ stage—number (\%) } \\
\hline NO & $409(100)$ & $757(100)$ & $1907(100)$ & $0(0)$ & 751 (58) & $1956(55)$ \\
\hline $\mathrm{N} 1$ & $0(0)$ & $0(0)$ & $0(0)$ & $5192(100)$ & $554(42)$ & $1605(45)$ \\
\hline
\end{tabular}

(62.7 (62.2 to 63.3$)$ vs 66.4 (66.2 to 66.6$)$ years) and other ethnic groups (65.6 (64.9 to 66.4) years; $\mathrm{p}<0.0001$ for both comparisons). Caucasians were older on average than all other ethnic groups $(\mathrm{p}=0.034)$. African Americans also had larger tumours on average than other ethnic groups (mean tumour diameter, 4.0 (3.9 to 4.1$)$ vs 3.8 ( 3.8 to 3.8$) \mathrm{cm} ; \mathrm{p}=0.0001)$. There was no significant difference in the age of patients whose tumours were located in the head, body or tail of the pancreas (data not shown). The average size of tumours located in the head of pancreas was significantly smaller than those in the body and those in the body were significantly smaller than those in the tail; (mean tumour diameter; 3.5 (3.5 to 3.6$)$ vs 4.3 (4.2 to 4.4$)$ vs 4.8 (4.7 to 4.9 ) $\mathrm{cm}$; $\mathrm{p}<0.0001)$.

Patients with well-differentiated cancers were significantly younger at diagnosis (mean age, 63.9 (63.3 to 64.5) years) than patients with cancers of higher neoplastic grade (moderately, poorly or undifferentiated; 66.3 (66.0 to 66.5$)$, 66.4 (66.1 to $66.7)$ and 65.3 (64.0 to 66.6) years; $\mathrm{p}<0.0001, \mathrm{p}<0.0001$ and $\mathrm{p}=0.043$; respectively). Patients with well-differentiated (mean tumour diameter, $3.7(3.6$ to 3.8$) \mathrm{cm}$ ) and moderatedifferentiated cancers $(3.6(3.6$ to 3.7$) \mathrm{cm})$ had smaller tumours on average than patients with poorly differentiated cancers (4.0 (4.0 to 4.1$) \mathrm{cm}$; $\mathrm{p}=0.0001$ for both). Patients with undifferentiated cancers had the largest tumours $(4.7(4.4$ to 5.0$) \mathrm{cm}$; $\mathrm{p}<0.0001)$.

\section{Relationships between patient age and tumour size, T stage and overall stage}

To estimate the time it takes for a small localised T1 to progress to higher $\mathrm{T}$ stages, we compared the average adjusted age of patients with localised or locally advanced cancers without distant metastases after adjusting for patient ethnicity, sex, tumour location and grade. Patients with stage I cancers (mean age, 64.8 adjusted years) were on average 2.3 (0.56 to 1.9) adjusted years younger $(p=0.0004)$ than patients with higher stage cancers (mean adjusted age 66.1 years). This was most evident when comparing patients with stage I versus stage IV tumours (age difference $=1.3$ adjusted years, (0.51 to 2.1), $\mathrm{p}=0.001)$. Among patients diagnosed with stage I, II or III cancers, the average adjusted age of patients with T3 or T4 tumours was significantly older than patients with T1 tumours (by 1.06 adjusted years for T3 vs T1, and 1.19 adjusted years for T4 vs T1, p=0.03 for both; table 3). Interestingly, this age/T stage relationship was not significant for patients with distant metastases (stage IV disease; see online supplementary tables). Therefore, when patients of all stages were compared, the average adjusted age of patients with T3 and T4 tumours was not significantly higher $(\sim 0.5$ adjusted years) than those with T1 tumours (see online supplementary tables). This lack of age difference among patients with stage IV disease may in part reflect how primary tumour size is measured in patients with metastatic 
Table 2 Mean (SD) of age at diagnosis, for all Surveillance, Epidemiology and End Results (SEER) participants, by stage and other clinical characteristics

\begin{tabular}{|c|c|c|c|c|c|c|c|}
\hline & All patients & Stage IA & Stage IB & Stage IIA & Stage IIB & Stage III & Stage IV \\
\hline \multicolumn{8}{|c|}{ Race-mean (SD) } \\
\hline Black & $62.7(11)$ & $61.2(10)$ & $58.9(11.3)$ & $63.8(10.5)$ & $61.8(10.5)$ & $64.5(11.3)$ & $63.4(11.4)$ \\
\hline Other & $65.6(12)$ & $60.3(11.5)$ & $65.1(11.4)$ & $64.8(11.7)$ & $65.7(11.6)$ & $67.4(12.1)$ & $66(12.7)$ \\
\hline White & $66.4(11.2)$ & $65.5(11)$ & $65.7(11.9)$ & $67.7(10.4)$ & $66.1(10.8)$ & $66.2(11.5)$ & $66.6(12)$ \\
\hline \multicolumn{8}{|c|}{ Sex-mean (SD) } \\
\hline Female & $66.8(11.5)$ & $64.7(11.7)$ & $63.9(12.7)$ & $67.4(10.7)$ & $66.7(10.9)$ & $67.1(11.9)$ & $67.3(12.3)$ \\
\hline Male & $65.2(11)$ & $64.8(10.5)$ & $65.9(11)$ & $66.8(10.6)$ & $64.7(10.8)$ & $65.1(11)$ & $65.1(11.6)$ \\
\hline \multicolumn{8}{|c|}{ Location-mean (SD) } \\
\hline Body & $66.2(11.5)$ & $63.6(11.1)$ & $65.4(12.4)$ & $67.8(10)$ & $65.2(11.5)$ & $66.2(11.5)$ & $66.7(11.6)$ \\
\hline Head & $66(11.1)$ & $65.2(11.2)$ & $65.5(11.1)$ & $67.1(10.4)$ & $65.7(10.8)$ & $66(11.4)$ & $66.2(11.9)$ \\
\hline Tail & $65.5(12.2)$ & $64.3(10.9)$ & $63.1(13.4)$ & $66.8(11.9)$ & $65.8(11.2)$ & $67.1(12.8)$ & $65.5(12.5)$ \\
\hline \multicolumn{8}{|c|}{ Grade-mean (SD) } \\
\hline G1 & $63.9(12.4)$ & $62.5(11.3)$ & $61.7(12.8)$ & $65.9(11.4)$ & $63.7(12.1)$ & 66 (11.6) & $63.5(13.7)$ \\
\hline G2 & $66.3(10.9)$ & $66.9(10.3)$ & $65.3(11.7)$ & $67.3(10.3)$ & $65.9(10.6)$ & $66.1(11.4)$ & $66.6(11.7)$ \\
\hline G3 & $66.4(11.2)$ & $66.2(11.4)$ & $67.7(10.7)$ & $67.4(10.6)$ & $66(10.8)$ & $66.1(11.6)$ & $66.4(11.7)$ \\
\hline G4 & $65.3(11.5)$ & $55.6(9.9)$ & $64.9(10.6)$ & $66.3(12.5)$ & $64.5(11)$ & $66.3(11.8)$ & $65.8(11.5)$ \\
\hline \multicolumn{8}{|c|}{ Size $(\mathrm{cm})$ - mean (SD) } \\
\hline$<2$ & $65.6(11.2)$ & $64.8(11.1)$ & (NA) & $66.8(11.1)$ & $65(11)$ & $66.4(11.6)$ & $67.3(11.8)$ \\
\hline $2-4$ & $66.2(11.1)$ & (NA) & $65.4(11.2)$ & $67.5(10.2)$ & $66(10.9)$ & $66.1(11.3)$ & $66.1(12)$ \\
\hline$>4$ & $65.7(11.6)$ & (NA) & $63.4(13.4)$ & $66.3(11.2)$ & $65.3(10.7)$ & $66(11.8)$ & $65.9(12)$ \\
\hline \multicolumn{8}{|c|}{ T Stage-mean (SD) } \\
\hline $\mathrm{T} 1$ & $65.1(11.4)$ & $64.8(11.1)$ & (NA) & (NA) & $64.3(11.8)$ & (NA) & $67.5(11.7)$ \\
\hline $\mathrm{T} 2$ & $65.9(11.9)$ & (NA) & $64.9(12)$ & (NA) & $64.9(11.4)$ & (NA) & $67.1(12.1)$ \\
\hline T3 & $66.1(11)$ & (NA) & (NA) & $67.1(10.6)$ & $65.8(10.8)$ & (NA) & $65.6(12.2)$ \\
\hline T4 & $65.9(11.5)$ & (NA) & (NA) & (NA) & (NA) & $66.1(11.5)$ & $65.6(11.6)$ \\
\hline \multicolumn{8}{|c|}{ N Stage-mean (SD) } \\
\hline NO & $66.6(11.4)$ & $64.8(11.1)$ & $64.9(12)$ & $67.1(10.6)$ & (NA) & $66.8(11.7)$ & $67.3(11.8)$ \\
\hline N1 & $65.4(11.2)$ & (NA) & (NA) & (NA) & $65.7(10.9)$ & $65.1(11.3)$ & $64.7(12.1)$ \\
\hline
\end{tabular}

pancreatic cancer (by imaging tests), whereas patients who underwent pancreatic resection had pathological measurements of their primary cancer. Pathological measurements of tumour size are generally more accurate than those measured by abdominal imaging. In addition, while primary tumour size measurements are probably good estimates of overall tumour burden (and therefore disease duration) in patients with localised or locally advanced pancreatic cancer, the same may not be true for patients with metastatic pancreatic cancer.

Table 3 Estimates from multiple linear regression models for age, adjusting for patient race, sex, tumour location and grade among patients with stage I or II disease (first column) and stage I, II or III disease (second column)

\begin{tabular}{lll}
\hline & Stages I, II & Stages I, II, III \\
\hline $\begin{array}{l}\text { Adjusted mean } \\
\text { age (years), T1 }\end{array}$ & 60.03 (58.56 to 61.5) & 60.68 (59.3 to 62.06) \\
$\begin{array}{l}\text { Age difference } \\
\text { (years): T2 vs T1 }\end{array}$ & $-0.01(-1.06$ to 1.05), $\mathrm{p}=0.99$ & $0.03(-1.03$ to 1.1$), \mathrm{p}=0.95$ \\
$\begin{array}{l}\text { Age difference } \\
\text { (years): T3 vs T1 }\end{array}$ & $0.95(0.01$ to 1.89), $\mathrm{p}=0.05$ & $1.06(0.11$ to 2$), \mathrm{p}=0.03$ \\
$\begin{array}{l}\text { Age difference } \\
\text { (years): T4 vs T1 }\end{array}$ & N.A. & $1.19(0.11$ to 2.27$), \mathrm{p}=0.03$ \\
\hline $\begin{array}{l}\text { Separate models were estimated for varying subgroups of patient stage of disease. } \\
\text { Values given are mean differences in ages by T stage, with } 95 \% \text { Cls. }\end{array}$
\end{tabular}

We therefore examined differences in the adjusted age of patients with localised or locally advanced disease according to their primary tumour T stage and size within and between different tumor, node, metastasis (TNM) stages. This analysis reveals some expected and some unexpected patterns in the patient age/ tumour size relationship. For example, although patients with the lowest stage cancers (stage IA and stage IB) were significantly younger than patients with stage IIA tumours, they were not younger than patients with stage IIB (node-positive) tumours (table 4, online supplementary table S4). This raises the possibility that biological differences between patients and their cancers that cause some cancers, but not others to spread to lymph nodes could obscure the overall relationship between increasing tumour size and stage and increasing patient age at diagnosis. For example, the age of patients with stage IIA cancers (T3N0M0) was significantly older than patients with stage IIB tumours (T3N1M0; by 1.39 adjusted years, $\mathrm{p}<0.0001$; see online supplementary table S3). Indeed, among patients whose node status was determined by pathological staging, those with node-positive cancers were significantly younger (by 0.6 adjusted years) than those with node negative disease $(p=0.014)$.

Since there are likely to be tumour and patient characteristics that result in patients having had node-positive rather than node-negative or metastatic rather than localised cancers at diagnosis, we focused on comparing the age of patients with tumours within specific TNM stages. In this analysis, there was a general trend within these stage subgroups for patients with larger tumours to be older than patients with smaller tumours, 
Table 4 Estimates from a multiple linear regression model for age, adjusting for patient race, sex, tumour location and grade, among patients with stage I, II or III disease only

\begin{tabular}{|c|c|}
\hline & Stages I to III only \\
\hline Adjusted mean age (years), stage IA & 60.77 (59.29 to 62.25$)$ \\
\hline Age difference (years): IB vs IA & $-0.15(-1.46$ to 1.17$), p=0.83$ \\
\hline Age difference (years): IIA, $\leq 2 \mathrm{~cm}$ vs IA & $1.51(-0.07$ to 3.08$), p=0.06$ \\
\hline Age difference (years): IIA, $2-4 \mathrm{~cm}$ vs IA & 2.09 (0.83 to 3.35$), p=0.001$ \\
\hline Age difference (years): $\| \mathrm{A},>4 \mathrm{~cm}$ vs IA & $1.09(-0.37$ to 2.56$), p=0.14$ \\
\hline Age difference (years): IIB, $\leq 2 \mathrm{~cm}$ vs IA & $-0.47(-1.81$ to 0.87$), p=0.49$ \\
\hline Age difference (years): IIB, 2-4 cm vs IA & $0.55(-0.6$ to 1.71$), p=0.35$ \\
\hline Age difference (years): IIB, $>4 \mathrm{~cm}$ vs IA & $-0.08(-1.31$ to 1.15$), p=0.9$ \\
\hline Age difference (years): III, $\leq 2 \mathrm{~cm}$ vs IA & $1.39(-1.08$ to 3.86$), p=0.27$ \\
\hline Age difference (years): III, 2-4 cm vs IA & $0.77(-0.62$ to 2.16$), p=0.28$ \\
\hline Age difference (years): III, $>4 \mathrm{~cm}$ vs IA & $1(-0.38$ to 2.38$), p=0.15$ \\
\hline
\end{tabular}

Values given are mean differences in ages by tumour size and stage of disease, with $95 \%$ Cls.

but the age differences were small (typically a few months) and often not statistically significant. Thus, there was no significant difference in the adjusted mean age of patients with stage IB versus stage IA tumours even though stage IB tumours were on average $2.5 \mathrm{~cm}$ diameter larger than IA tumours (table 4). There was also no significant adjusted age difference between patients with stage IIA tumours larger than $2 \mathrm{~cm}$ and those with tumours $\leq 2 \mathrm{~cm}$ ( 0.31 adjusted years, $\mathrm{p}=0.63$; table 5). Among patients with stage IIB tumours, the mean adjusted age of patients with T3 tumours was 0.79 years older than it was for patients with T1 or T2 tumours $(p=0.06$; table 6$)$. Among patients with stage IIB tumours, those with T3 tumours of $>2 \mathrm{~cm}$ trended towards being older than patients with T3 tumours $\leq 2 \mathrm{~cm}$ (by 0.84 adjusted-years, $\mathrm{p}=0.09$ ), and were
Table 6 Estimates from a multiple linear regression model for age, adjusting for patient race, sex, tumour location and grade, among patients with stage IIB disease only

\begin{tabular}{lc}
\hline & Stage IIB Only \\
\hline Adjusted mean age (years), stage IIB, T1 & $59.77(57.42$ to 62.13$)$ \\
Age difference (years): stage IIB, T2 vs T1 & $0.49(-1.33$ to 2.31$), \mathrm{p}=0.6$ \\
Age difference (years): stage IIB, T3 vs T1 & $1.17(-0.48$ to 2.81$), \mathrm{p}=0.16$ \\
Adjusted mean age (years), stage IIB, T1/T2 & $60.15(58.27$ to 62.03) \\
Age difference (years): stage IIB, T3 vs T1/T2 & $0.79(-0.03$ to 1.61$), \mathrm{p}=0.06$ \\
\hline Values given are mean differences in ages by tumour size, with $95 \% \mathrm{Cls}$.
\end{tabular}

significantly older than those with T1 and T2 tumours $(0.83$ adjusted years, $\mathrm{p}=0.05$; table 5 ). When we compared patients by the size of their primary tumour rather than its $\mathrm{T}$ stage, similar trends were noted: among patients with stage IIB tumours, those with tumours of $2-4 \mathrm{~cm}$ diameter were significantly older on average than patients with tumours $\leq 2 \mathrm{~cm}(1.02$ adjusted years, $\mathrm{p}=0.02$ ), although patients with $>4 \mathrm{~cm}$ tumours were only 0.39 adjusted years older than patients with tumours $\leq 2 \mathrm{~cm}(\mathrm{p}=0.45$; table 4$)$.

\section{DISCUSSION}

In this study we have calculated differences in the average age at diagnosis of patients with pancreatic cancers of different sizes and stages after adjusting for cofactors that are associated with age at diagnosis. These age differences provide an estimate of the average time it takes pancreatic cancers to grow within and through different stages of the disease. As a representative example, we found that among patients diagnosed with localised or locally advanced disease those diagnosed with T3 or T4 pancreatic cancers were 1.06 and 1.19 adjusted years older,

Table 5 Estimates from a multiple linear regression model for age, adjusting for patient race, sex, tumour location and grade, among patients with stage I and II disease only

\begin{tabular}{|c|c|}
\hline & Stages I and II only \\
\hline Adjusted mean age (years), stage I & 60.03 (58.71 to 61.35) \\
\hline Age difference (years): stage IIA, $\leq 2 \mathrm{~cm}$ vs stage I & $1.48(0.17$ to 2.79$), p=0.03$ \\
\hline Age difference (years): stage IIA, $>2 \mathrm{~cm}$ vs stage I & 1.79 (0.96 to 2.63$), p<0.0001$ \\
\hline Age difference (years): stage IIB, T1 and T2 vs stage I & $-0.41(-1.39$ to 0.58$), p=0.42$ \\
\hline Age difference (years): stage IIB, $T 3, \leq 2 \mathrm{~cm}$ vs stage I & $-0.41(-1.52$ to 0.7$), p=0.47$ \\
\hline Age difference (years): stage IIB, $T 3,>2 \mathrm{~cm}$ vs stage I & $0.43(-0.31$ to 1.17$), p=0.26$ \\
\hline Adjusted mean age (years), stage $\| A, \leq 2 \mathrm{~cm}$ & 61.51 (59.82 to 63.21) \\
\hline Age difference (years): stage $\| \mathrm{A},>2 \mathrm{~cm}$ vs stage $\| \mathrm{A}, \leq 2 \mathrm{~cm}$ & $0.31(-0.95$ to 1.57$), p=0.63$ \\
\hline Age difference (years): stage IIB, $\mathrm{T} 1$ and $\mathrm{T} 2$ vs stage $I I A, \leq 2 \mathrm{~cm}$ & $-1.89(-3.26$ to -0.52$), p=0.007$ \\
\hline Age difference (years): stage $\| \mathrm{B}, \mathrm{T} 3, \leq 2 \mathrm{~cm}$ vs stage $\| \mathrm{A}, \leq 2 \mathrm{~cm}$ & $-1.89(-3.34$ to -0.44$), p=0.01$ \\
\hline Age difference (years): stage IIB, $T 3,>2 \mathrm{~cm}$ vs stage IIA, $\leq 2 \mathrm{~cm}$ & $-1.06(-2.25$ to 0.14$), p=0.08$ \\
\hline Adjusted mean age (years), stage $I I A,>2 \mathrm{~cm}$ & $61.82(60.44$ to 63.21$)$ \\
\hline Age difference (years): stage IIB, T1 and T2 vs stage IIA, $>2 \mathrm{~cm}$ & $-2.2(-3.12$ to -1.28$), p<0.0001$ \\
\hline Age difference (years): stage $\| \mathrm{B}, \mathrm{T} 3, \leq 2 \mathrm{~cm}$ vs stage $\| \mathrm{A},>2 \mathrm{~cm}$ & $-2.2(-3.25$ to -1.15$), p<0.0001$ \\
\hline Age difference (years): stage IIB, $T 3,>2 \mathrm{~cm}$ vs stage $\| \mathrm{A},>2 \mathrm{~cm}$ & $-1.37(-2.01$ to -0.73$), p<0.0001$ \\
\hline Adjusted mean age (years), stage $I I B, T 1$ and $T 2$ & 59.62 (58.16 to 61.08$)$ \\
\hline Age difference (years): stage IIB, T3, $\leq 2 \mathrm{~cm}$ vs stage IIB, T1 and T2 & $0(-1.17$ to 1.17$), p=1$ \\
\hline Age difference (years): stage IIB, $\mathrm{T} 3,>2 \mathrm{~cm}$ vs stage IIB, $\mathrm{T} 1$ and $\mathrm{T} 2$ & $0.83(0.01$ to 1.66$), p=0.05$ \\
\hline Adjusted mean age (years), stage $\|(B, T 3, \leq 2 \mathrm{~cm}$ & 59.62 (58.04 to 61.2) \\
\hline Age difference (years): stage IIB, $\mathrm{T} 3,>2 \mathrm{~cm}$ vs stage IIB, $\mathrm{T} 3, \leq 2 \mathrm{~cm}$ & $0.84(-0.12$ to 1.8$), p=0.09$ \\
\hline Adjusted mean age (years), stage $\| I B, T 3,>2 \mathrm{~cm}$ & 60.46 (59.11 to 61.8$)$ \\
\hline
\end{tabular}

Values given are mean differences in ages by tumour size, with $95 \% \mathrm{Cls}$. 
respectively (13-14 months), than patients with T1 tumours $(p=0.03$; table 3$)$. Such small age differences between patients with high versus low $\mathrm{T}$ stage cancers are in contrast with estimates derived from molecular clock data ${ }^{17}$ and are consistent with the hypothesis that once a pancreatic cancer is detectable by diagnostic tests, its growth and progression to more advanced stage disease is rapid. It should be noted while that our analysis calculated an average time to progression through different TNM stages, there is likely to be significant heterogeneity in the rate of progression of pancreatic cancers in individual patients.

Evidence that pancreatic cancers rapidly grow through its clinical stages has implications for efforts at detecting pancreatic cancers while it is still at its lowest stage. Ideally, screen-detected cancers would be smaller than the average-sized stage I pancreatic cancer in the SEER database, and so theoretically would have a longer average time-to-progression. There have been only a few screen-detected pancreatic cancers reported in the literature, including cases detected at baseline screening, ${ }^{1-9}{ }^{11}$ and while most of these screen-detected cancers have been resectable cancers, ${ }^{9}$ few have been stage I cancers, and some were not detected until they were at an advanced stage. Until pancreatic screening programmes have identified more screen-detected pancreatic cancers and understand the challenges of detecting these earliest stage cancers, our results point to the possibility that many pancreatic cancers diagnosed by pancreatic screening will have progressed to advanced stage even when affected patients have undergone regular surveillance unless screening can identify the precancerous lesions likely to progress to invasive cancer in these patients and treat these lesions before they progress to invasive cancer. Better screening tests that can reliably detect very small T1 cancers $(<1 \mathrm{~cm}$, or better yet $<5 \mathrm{~mm}$ ), and if possible PanIN-3 (carcinoma in situ) would minimise the chance of having patients progressing to advanced stage disease while under surveillance. Current screening programmes emphasise the detection and management of precancerous cystic lesions, because these are the most common lesions detected by current screening tests and removing them provides an opportunity to prevent the development of pancreatic cancer. Resecting the pancreas in these individuals will also remove associated PanIN. Another reason why the focus on identifying and treating pancreatic precursor lesions is important is that efforts focused solely on detecting small T1 primary pancreatic cancers (those $<1 \mathrm{~cm}$ ) may be inadequate because many T1-stage cancers have spread beyond the pancreas. In our SEER sample, only 179 of 13131 patients (1.4\%) had pancreatic cancers with a diameter of $\leq 1 \mathrm{~cm}, 55$ of these $179(30.1 \%)$ had node-positive disease and $18(10.1 \%)$ had distant metastases. These data predict that screening protocols that continue surveillance until a pancreatic mass emerges and surgical intervention is undertaken may often fail to prevent death from pancreatic cancer. To increase the numbers of patients diagnosed with stage I pancreatic cancers more high-risk individuals need to undergo pancreatic screening and pancreatic screening programmes need to have well-validated diagnostic tests that can reliably detect carcinoma in situ and very small $(<5 \mathrm{~mm})$ pancreatic cancers.

Expanding pancreatic screening to other at-risk populations might help increase the proportion of patients who had earlystage subcentimetre pancreatic cancers, particularly if better screening tests were developed, ${ }^{14} 1525$ but long-term studies are still needed to evaluate the utility of screening the 'high-risk' populations with the appropriate age, family history and/or gene mutation status where screening is currently recommended. ${ }^{11}$ The benefits of pancreatic screening would likely improve if we were able to better estimate pancreatic cancer risk in the general population $^{26}$ and in families affected by the disease. ${ }^{27}$

Our analysis also confirmed prior associations between pancreatic cancer risk factors and age at diagnosis. Thus, it has been reported that African Americans are diagnosed with pancreatic cancer at an earlier age than other ethnic groups, ${ }^{28-30}$ as are individuals who smoke. ${ }^{31} 32$ The prevalence of known pancreatic cancer risk factors in African Americans versus Caucasians does not explain the earlier average age of pancreatic cancer diagnosis in African Americans. ${ }^{30}$ It has also been shown that cancers in the tail are generally larger than those in the head of the pancreas. $^{33}$ We also found that larger primary pancreatic ductal adenocarcinomas were more likely to be poorly differentiated. There is evidence that tumour hypoxia which is likely to be greater in larger tumours contributes to the differentiation state of the tumour. ${ }^{34}$

We were not able to identify any significant differences in the age of patients with different sized stage I cancers, perhaps because of the small number of patients available for analysis with stage I pancreatic cancers. Another limitation of our study is that although we adjusted for factors in our data set associated with patient age and tumour size at diagnosis, other risk factors for pancreatic cancer development not in the SEER data set may influence the age of pancreatic cancer diagnosis that if accounted for could provide more reliable estimates of the average age of patients at diagnosis and the size and stage of their tumours. One limitation to using tumour size as a measurement of pancreatic cancer progression is that such measurements do not take into account factors such as the contribution of tumour stroma to tumour size and the limitation of tumour size measurements of resection margin-positive cancers. Although the SEER database is a valuable resource, it does not collect data on resection margin status. It is possible that primary tumour size estimates that accounted for margin status would be more accurate than those without. Incomplete ascertainment can be a concern for SEER-based studies and for cross-sectional studies in general, ${ }^{35} 36$ particularly studies of outcomes of patients assigned to different treatments. ${ }^{37}$ Our inclusion criteria only required cases have a complete set of pathological staging and demographic so we would not expect there to be significant bias related to the exclusion of cases without a complete set of data.

In conclusion, we find using the adjusted average ages of patients with localised or locally advanced pancreatic cancer at diagnosis that disease progression is rapid, with an average estimated time of 14 months for a T1 pancreatic cancer to progress to the $\mathrm{T} 4$ stage.

\section{Author affiliations}

${ }^{1}$ Department of Pathology, The Sol Goldman Pancreatic Cancer Research Center, The Johns Hopkins University School of Medicine, Baltimore, Maryland, USA

${ }^{2}$ Department of Oncology, The Sol Goldman Pancreatic Cancer Research Center, The Johns Hopkins University School of Medicine, Baltimore, Maryland, USA

${ }^{3}$ Department of Surgery, The Sol Goldman Pancreatic Cancer Research Center, The Johns Hopkins University School of Medicine, Baltimore, Maryland, USA

${ }^{4}$ Department of Medicine, The Sol Goldman Pancreatic Cancer Research Center, The Johns Hopkins University School of Medicine, Baltimore, Maryland, USA

Contributors MG was involved in study concept and design, drafting of the manuscript, obtained funding and study supervision. JY, MdM, CLW was involved in acquisition of data. ALB, JY were involved in statistical analysis. JY, ALB, MdM,

CLW, MG were involved in interpretation of data revision of manuscript. All authors were involved in the interpretation of the data; revision of the manuscript; and the decision to submit the manuscript for publication.

Funding This work was supported by Susan Wojcicki and Dennis Troper, $\mathrm{NIH}$ grants (CA62924 and R01CA176828), the Pancreatic Cancer Action Network-American Association for Cancer Research (AACR) Research Acceleration Grant. 
Competing interests None.

Provenance and peer review Not commissioned; externally peer reviewed.

Open Access This is an Open Access article distributed in accordance with the Creative Commons Attribution Non Commercial (CC BY-NC 4.0) license, which permits others to distribute, remix, adapt, build upon this work non-commercially, and license their derivative works on different terms, provided the original work is properly cited and the use is non-commercial. See: http://creativecommons.org/ licenses/by-nc/4.0/

\section{REFERENCES}

1 Canto MI, Hruban RH, Fishman EK, et al. Frequent detection of pancreatic lesions in asymptomatic high-risk individuals. Gastroenterology 2012;142:796-804.

2 Ludwig E, Olson SH, Bayuga S, et al. Feasibility and yield of screening in relatives from familial pancreatic cancer families. Am J Gastroenterol 2011;106:946-54.

3 Verna EC, Hwang C, Stevens PD, et al. Pancreatic cancer screening in a prospective cohort of high-risk patients: a comprehensive strategy of imaging and genetics. Clin Cancer Res 2010;16:5028-37.

4 Langer $\mathrm{P}$, Kann $\mathrm{PH}$, Fendrich $\mathrm{V}$, et al. Five years of prospective screening of high-risk individuals from families with familial pancreatic cancer. Gut 2009;58:1410-18.

5 Canto Ml, Goggins M, Hruban RH, et al. Screening for early pancreatic neoplasia in high-risk individuals: a prospective controlled study. Clin Gastroenterol Hepatol 2006:4:766-81.

6 Canto Ml, Goggins M, Yeo CJ, et al. Screening for pancreatic neoplasia in high-risk individuals: an EUS-based approach. Clin Gastroenterol Hepatol 2004;2:606-21.

7 Brentnall T, Bronner M, Byrd D, et al. Early diagnosis and treatment of pancreatic dysplasia in patients with a family history of pancreatic cancer. Ann Intern Med 1999;131:247-55.

8 Poley JW, Kluijt I, Gouma DJ, et al. The yield of first-time endoscopic ultrasonography in screening individuals at a high risk of developing pancreatic cancer. Am J Gastroenterol 2009;104:2175-81.

9 Vasen $H F$, Wasser $M$, van Mil A, et al. Magnetic resonance imaging surveillance detects early-stage pancreatic cancer in carriers of a p16-leiden mutation. Gastroenterology 2011;140:850-6.

10 Brune K, Abe T, Canto M, et al. Multifocal neoplastic precursor lesions associated with lobular atrophy of the pancreas in patients having a strong family history of pancreatic cancer. Am J Surg Pathol 2006;30:1067-76.

11 Canto Ml, Harinck F, Hruban RH, et al. International consensus recommendations on the management of patients with increased risk for familial pancreatic cancer (The Cancer of the Pancreas Screening (CAPS) consortium summit). Gut 2013;62:339-47.

12 Tanaka M, Fernandez-Del Castillo C, Adsay V, et al. International consensus guidelines 2012 for the management of IPMN and MCN of the pancreas. Pancreatology 2012;12:183-97.

13 Norris AL, Roberts NJ, Jones S, et al. Familial and sporadic pancreatic cancer share the same molecular pathogenesis. Fam Cancer 2014. In press.

14 Lennon AM, Wolfgang CL, Canto Ml, et al. The early detection of pancreatic cancer: what will it take to diagnose and treat curable pancreatic neoplasia? Cancer Res 2014;74:3381-9.

15 Kanda M, Sadakari Y, Borges M, et al. Mutant TP53 in duodenal samples of pancreatic juice from patients with pancreatic cancer or high-grade dysplasia. Clin Gastroenterol Hepatol 2013;11:719-30.

16 Khashab MA, Yong E, Lennon AM, et al. EUS is still superior to multidetector computerized tomography for detection of pancreatic neuroendocrine tumors. Gastrointest Endosc 2011;73:691-6.
17 Yachida S, Jones S, Bozic I, et al. Distant metastasis occurs late during the genetic evolution of pancreatic cancer. Nature 2010;467:1114-17.

18 Ferrone $C R$, Pieretti-Vanmarcke R, Bloom JP, et al. Pancreatic ductal adenocarcinoma: long-term survival does not equal cure. Surgery 2012; 152:543-9.

19 Winter JM, Cameron JL, Campbell KA, et al. 1423 pancreaticoduodenectomies for pancreatic cancer: a single-institution experience. J Gastrointest Surg 2006:10:1199-210; discussion 1210-1191.

20 de Jong MC, Li F, Cameron JL, et al. Re-evaluating the impact of tumor size on survival following pancreaticoduodenectomy for pancreatic adenocarcinoma. J Surg Oncol 2011;103:656-62.

21 Honda O, Johkoh T, Sekiguchi J, et al. Doubling time of lung cancer determined using three-dimensional volumetric software: comparison of squamous cell carcinoma and adenocarcinoma. Lung Cancer 2009;66:211-17.

22 Nishida K, Kaneko T, Yoneda M, et al. Doubling time of serum CA 19-9 in the clinical course of patients with pancreatic cancer and its significant association with prognosis. J Surg Oncol 1999;71:140-6.

23 Gerlee P. The model muddle: in search of tumor growth laws. Cancer Res 2013:73:2407-11.

24 Edwards BK, Brown ML, Wingo PA, et al. Annual report to the nation on the status of cancer, 1975-2002, featuring population-based trends in cancer treatment. J Natl Cancer Inst 2005;97:1407-27.

25 Kanda M, Knight S, Topazian M, et al. Mutant GNAS detected in duodenal collections of secretin-stimulated pancreatic juice indicates the presence or emergence of pancreatic cysts. Gut 2013;62:1024-33.

26 Klein AP, Lindstrom S, Mendelsohn JB, et al. An absolute risk model to identify individuals at elevated risk for pancreatic cancer in the general population. PLOS ONE 2013:8:e72311.

27 Klein AP. Identifying people at a high risk of developing pancreatic cancer. Nat Rev Cancer 2013;13:66-74.

28 Chang KJ, Parasher G, Christie C, et al. Risk of pancreatic adenocarcinoma: disparity between African Americans and other race/ethnic groups. Cancer 2005;103:349-57.

29 Wray CJ, Castro-Echeverry E, Silberfein EJ, et al. A multi-institutional study of pancreatic cancer in Harris County, Texas: race predicts treatment and survival. Ann Surg Oncol 2012;19:2776-81.

30 Arnold LD, Patel AV, Yan Y, et al. Are racial disparities in pancreatic cancer explained by smoking and overweight/obesity? Cancer Epidemiol Biomarkers Prev 2009;18:2397-405.

31 James TA, Sheldon DG, Rajput A, et al. Risk factors associated with earlier age of onset in familial pancreatic carcinoma. Cancer 2004;101:2722-6.

32 Anderson MA, Zolotarevsky E, Cooper KL, et al. Alcohol and tobacco lower the age of presentation in sporadic pancreatic cancer in a dose-dependent manner: a multicenter study. Am J Gastroenterol 2012;107:1730-9.

33 Sohn TA, Yeo CJ, Cameron JL, et al. Resected adenocarcinoma of the pancreas-616 patients: results, outcomes, and prognostic indicators. J Gastrointest Surg 2000:4:567-79.

34 Axelson $\mathrm{H}$, Fredlund $\mathrm{E}$, Ovenberger $\mathrm{M}$, et al. Hypoxia-induced dedifferentiation of tumor cells - a mechanism behind heterogeneity and aggressiveness of solid tumors. Semin Cell Dev Biol 2005;16:554-63.

35 Bull K, Spiegelhalter DJ. Survival analysis in observational studies. Stat Med 1997;16:1041-74.

36 Cain KC, Harlow SD, Little RJ, et al. Bias due to left truncation and left censoring in longitudinal studies of developmental and disease processes. Am J Epidemiol 2011;173:1078-84.

37 Park HS, Lloyd S, Decker RH, et al. Limitations and biases of the Surveillance, Epidemiology, and End Results database. Curr Probl Cancer 2012;36:216-24. 